Draft version April 21, 2022

Preprint typeset using $\mathrm{LATEX}_{\mathrm{E}}$ style emulateapj v. 5/14/03

\title{
MASSIVE SATELLITES OF CLOSE-IN GAS GIANT EXOPLANETS
}

\author{
Timothy A. Cassidy ${ }^{1}$, Rolando Mendez ${ }^{2}$, Phil Arras ${ }^{2}$, Robert E. Johnson ${ }^{1,2}$, Michael F. Skrutskie ${ }^{2}$ \\ Draft version April 21, 2022
}

\begin{abstract}
We study the orbits, tidal heating and mass loss from satellites around close-in gas giant exoplanets. The focus is on large satellites which are potentially observable by their transit signature. We argue that even Earth-size satellites around hot Jupiters may be immune to destruction by orbital decay; detection of such a massive satellite would strongly constrain theories of tidal dissipation in gas giants, in a manner complementary to orbital circularization. The star's gravity induces significant periodic eccentricity in the satellite's orbit. The resulting tidal heating rates, per unit mass, are far in excess of Io's and dominate radioactive heating out to planet orbital periods of months for reasonable satellite tidal $Q$. Inside planet orbital periods of about a week, tidal heating can completely melt the satellite. Lastly, we compute an upper limit to the satellite mass loss rate due to thermal evaporation from the surface, valid if the satellite's atmosphere is thin and vapor pressure is negligible. Using this upper limit, we find that although rocky satellites around hot Jupiters with orbital periods less than a few days can be significantly evaporated in their lifetimes, detectable satellites suffer negligible mass loss at longer orbital periods.

Subject headings:
\end{abstract}

\section{INTRODUCTION}

The high photometric precision of the Hubble Space Telescope allows interesting constraints to be placed on the existence of massive satellites orbiting transiting exoplanets. Two systems have been searched so far, HD 209458b (Brown et al. 2001) and HD 189733b (Pont et al. 2007). From the transit lightcurve and timing, upper limits of roughly an Earth radius and mass have been placed on hypothetical satellites around these two planets. A number of studies have discussed detection techniques (Barnes \& Fortnev 2004; Dovle \& Deeg 2004; Han \& Han 2002; Kipping 2009a, b; Sartoretti \& Schneider 1999; Simon et al. 2007; Szabó et al. 2006). This paper is concerned with the orbits and physical structure of these hypothetical moons.

With the launch of the Kepler satellite Borucki et al. 2004), 10 5 stars will be monitored with photometric precision sufficient to detect Earth size objects. The estimated hundreds of hot Jupiters (gas giant planets with orbital periods less than 1 week) to be found by Kepler will provide a large sample to investigate the existence of Earth-size satellites (Szabó et al. 2006).

Johnson \& Huggins (2006) note another possible observational manifestation of satellites. Interaction between a satellite and the planet's magnetosphere may give rise to an extended gaseous torus orbiting the planet, as in the Jupiter-Io system. Material stripped from the satellite's surface and atmosphere would contribute to the transmission spectrum of the planet. It has been suggested (Vidal-Madiar et al. 2003) that absorption in the upper atmosphere of HD 209458b (Ballester et al. 2007; Charbonneau et al. 2002; Redfield et al. 2008; Vidal-Madiar et al. 2003, 2004) is due to a high rate of atmospheric escape which increases the density at large

\footnotetext{
${ }^{1}$ Engineering Physics Program, University of Virginia Charlottesville, VA 22904-4325

2 Department of Astronomy, University of Virginia P.O. Box 400325, Charlottesville, VA 22904-4325
}

radii. The plasma torus model is an alternative explanation for such absorption high in the atmosphere.

There has been interest recently in the properties of satellites around exoplanets, mainly focused on the habitability of moons (e.g. Revnolds et al. 1987; Scharf 2006; Williams et al. 1997) around planets at large orbital separation from the star. Our focus in this paper is on planets to be found with Kepler, hence we restrict our attention on close-in planets for which the transit probability is substantially higher. As we will show, moons in such systems will be subjected to intense tidal heating, with consequences for the moon's structure, evaporation rate, and interaction with its surroundings.

Two recent studies of the orbital stability of satellites are important in this work. Domingos et al. (2006) investigated orbital stability for point mass star, planet and satellite, for a range of eccentricity of both the planet and satellite. They point out that, for small planetary eccentricity, satellite orbits are stable for $a_{s} \lesssim 0.5 a_{H}$, where $a_{s}$ is the semi-major axis of the satellite around the planet and $a_{H}$ is the planet's Hill radius. As we will show, the satellite orbits have large periodic eccentricity induced by the star as the stability limit $a_{s} \simeq 0.5 a_{H}$ is approached. Hence, while Io's eccentricity and tidal heating are due to interaction with Jupiter and the other Galilean satellites, moons of close in exoplanets have a large periodic eccentricity due to stellar forcing. We show that this periodic eccentricity leads to large tidal heating for systems close to the parent star. This tidal heating may have a significant impact on the structure of the satellite, inducing melting of the interior and perhaps reinforcing dynamo generation of magnetic fields in the core.

As pointed out by Barnes \& O'Brien (2002), such close-in moons, orbiting slowly rotating planets synchronized to the star, are subject to orbital decay. The tide raised on the planet by the moon acts to spin up the planet while shrinking the moons orbit, eventually leading to the moon impacting the planet. The orbital decay rate is sensitive to the tidal $Q_{p}$ of the gas giant planet. 
Barnes \& O'Brien (2002) used $Q_{p}=10^{5}$, the estimate for the Jupiter-Io tidal interaction, leading to the constraint that hot Jupiters cannot have moons larger than $\sim 10^{-4} M_{\oplus}$, where $M_{\oplus}$ is the Earth's mass. Such tiny moons would be undetectable by the transit method. However, as we discuss, the appropriate value of $Q_{p}$ may be larger by a factor $\sim 10^{8}$, allowing stable orbits for potentially detectable moons with radii $\gtrsim R_{\oplus}$.

Little attention has been given to the formation scenario of the massive satellites discussed in this paper. Canup \& Ward (2006) note that the outer planets of the Solar System contain only a fraction $10^{-4}$ of the planet's mass in the satellite systems. If applied to extrasolar planets, this phenomenological scaling implies an upper limit for the satellite mass of $\lesssim M_{\oplus} / 30$ which would be undetectable by the transit method. An alternative possibility is for the gas giant to capture a terrestrial planet into a bound orbit.

Satellite orbits in the restricted 3 body problem are discussed in section 2. Orbital decay due to the tide raised in the planet by the satellite is discussed in section 3. Tidal heating of the satellite due to the non-circular orbit and its consequences for internal structure are discussed in section 4. Coupled tidal heating and satellite orbital evolution are described in section 5. The rate of evaporative mass loss from the satellite is discussed in section 6 and conclusions are stated in section 7 .

\section{SATELLITE ORBITS}

In this section we review the moon's orbit, treating moon, planet and star as point masses. This problem is referred to as the "main problem in lunar theory" (Brouwer \& Clemence 1961), and considerable analytic progress can be made. We will give a simple derivation of the satellite's orbit, as needed to compute tidal heating, for the case where the planet's orbit around the star is circular (the "circular restricted 3-body problem") and the "unperturbed" orbit of the satellite is circular. That is, we will compute the small deviations from a circular orbit due to the gravity of the star.

We consider star, planet and satellite of masses $M_{*} \gg$ $M_{p} \gg M_{s}$, respectively. We will ignore the influence of the satellite on the star-planet orbit, treating it as Keplerian with semi-major axis $a_{p}$ and eccentricity $e_{p}$. The mean angular motion of the planet's orbit is $n_{p} \simeq\left(G M_{*} / a_{p}^{3}\right)^{1 / 2}=2 \pi / P_{p}$, where $P_{p}$ is the planet's orbital period. The satellite's orbit around the planet can be treated as nearly Keplerian, with semi-major axis $a_{s}$, eccentricity $e_{s}$ and mean motion $n_{s} \simeq\left(G M_{p} / a_{s}^{3}\right)^{1 / 2}=$ $2 \pi / P_{s}$.

We work in a reference frame with origin at the center of the planet and non-rotating axes fixed with respect to distant observers. In this reference frame the star only contributes a tidal force. Cylindrical coordinates $\left(r_{s}, \phi_{s}\right)$ and $\left(r_{*}, \phi_{*}\right)$ are used for the positions of the satellite and star, respectively. The orbits are assumed coplanar for simplicity. The equations of motion for the satellite are then

$$
\begin{aligned}
& \ddot{r}_{s}=\frac{\ell_{s}^{2}}{r_{s}^{3}}-\frac{G M_{p}}{r_{s}^{2}}+\frac{\partial \mathcal{R}}{\partial r_{s}} \\
& \dot{\phi}_{s}=\frac{\ell_{s}}{r_{s}^{2}}
\end{aligned}
$$

$$
\dot{\ell}_{s}=\frac{\partial \mathcal{R}}{\partial \phi_{s}}
$$

where $\ell_{s}$ is the satellite's orbital angular momentum per unit mass and $\mathcal{R}=\mathcal{R}\left(r_{s}, \phi_{s}, r_{*}, \phi_{*}\right)$ is the negative of the tidal gravitational potential from the star. We can simplify the form of $\mathcal{R}$ using the fact that $r_{s} \ll r_{*}$ to find the leading order result in $r_{s} / r_{*}$ (Brouwer \& Clemence 1961),

$$
\mathcal{R}=\frac{G M_{*} r_{s}^{2}}{2 r_{*}^{3}}\left(3 \cos ^{2}\left[\phi_{s}-\phi_{*}\right]-1\right) .
$$

Given an orbit for the planet-star system, $r_{*}(t)$ and $\phi_{*}(t)$, eq.11, 2 and 3 can be integrated in time to find the orbit of the satellite.

The parameter space for stable satellite orbits is strongly constrained. For close-in planets, the planetary radius $R_{p}$ can be a large fraction of the planet's Hill radius, $a_{H}=a_{p}\left(M_{p} / 3 M_{*}\right)^{1 / 3}$, implying that all of its bound satellite orbits are significantly perturbed by the star's gravity. Domingos et al. (2006) found stable orbits for $a_{s} \lesssim 0.49 a_{H}\left(1.0-1.0 e_{p}-0.27 e_{s}\right)$. In terms of the orbital periods, the stability limit is $P_{s} \lesssim P_{p} / 5$ for $e_{p}, e_{s} \ll 1$. The dimensionless strength of the disturbing potential is then $\mathcal{R} /\left(G M_{p} / a_{s}\right) \sim\left(n_{p} / n_{s}\right)^{2}=$ $\left(P_{s} / P_{p}\right)^{2} \leq 1 / 25$. Ignoring the cohesive strength, the moon must also orbit outside the planet's Roche radius at $a_{\text {Roche }}=3^{1 / 3} R_{p}\left(M_{p} R_{s}^{3} / M_{s} R_{p}^{3}\right)^{1 / 3}$, where $R_{p}$ is the radius of the planet. For gas giants with mean density $\rho_{p}=$ $3 M_{p} /\left(4 \pi R_{p}^{3}\right) \sim 1 \mathrm{~g} \mathrm{~cm}^{-3}$ and terrestrial satellites with mean density $\rho_{s}=3 M_{s} /\left(4 \pi R_{s}^{3}\right) \sim 5 \mathrm{~g} \mathrm{~cm}^{-3}$, the Roche radius is at or below the planets surface. Hence we can consider the satellite disrupted if it hits the planet's surface. In summary, satellite orbits are bounded at short orbital periods by the planet's surface and at long orbital periods by the stability constraint, giving the range of possible orbital periods $2 \pi\left(R_{p}^{3} / G M_{p}\right)^{1 / 2} \lesssim P_{s} \lesssim P_{p} / 5$, for $e_{s}, e_{p} \ll 1$.

The derivation in Brouwer \& Clemence (1961) uses the disturbing function formalism and computes the variation of the orbital elements $a_{s}, e_{s}$, etc rather than $r_{s}$ and $\phi_{s}$ directly. Here we give a simpler derivation of the variation involving $r_{s}$ and $\phi_{s}$. We consider a circular planetary orbit with $r_{*}=a_{p}$ and $\phi_{*}=n_{p} t$, and a satellite orbit described by a circular orbit plus small perturbations induced by the star. In detail, we write

$$
\begin{aligned}
r_{s}(t) & =a_{s}+\delta r_{s}(t) \\
\phi_{s}(t) & =\phi_{s 0}+n_{s} t+\delta \phi_{s}(t) \\
\ell_{s}(t) & =a_{s}^{2} n_{s}+\delta \ell_{s}(t) .
\end{aligned}
$$

Here $\phi_{s 0}$ is a constant phase. Plugging eq 5, 6 and 7 into eq,1, 2 and 3, the equations are satisfied at leading order. Eq3 can be immediately integrated to yield

$$
\frac{\delta \ell_{s}(t)}{\ell_{s}}=\frac{3}{4}\left(\frac{n_{p}}{n_{s}}\right)^{2} \cos \left[2\left(n_{s}-n_{p}\right) t+2 \phi_{s 0}\right],
$$

where we have approximated $n_{p} \ll n_{s}$ to simplify the expression. Plugging eq, 8 into eq, we find the following forced harmonic oscillator equation for $\delta r$,

$$
\ddot{\delta r_{s}}+n_{s}^{2} \delta r_{s}=n_{p}^{2} a_{s}\left(\frac{1}{2}+3 \cos \left[2\left(n_{s}-n_{p}\right) t+2 \phi_{s 0}\right]\right)(.9)
$$




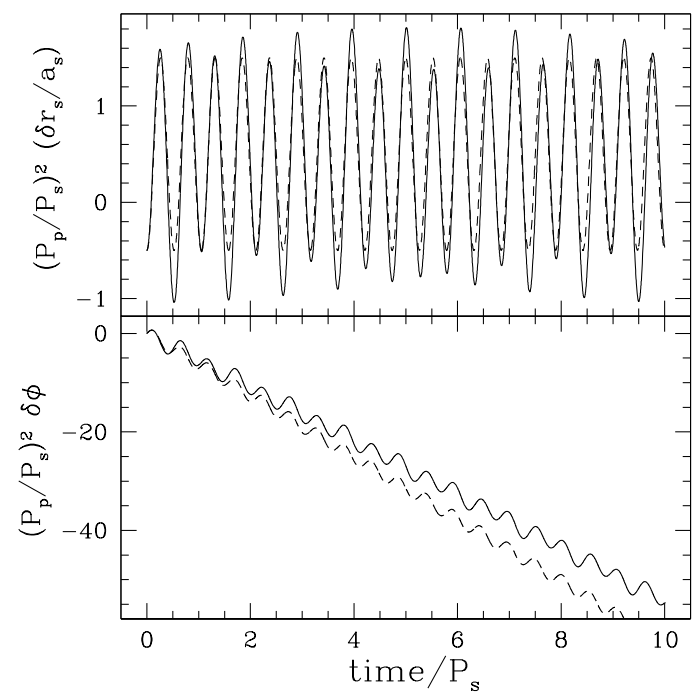

FIG. 1.- Perturbations to the radius (upper panel) and longitude (lower panel) due to the stellar gravitational field. Here $\delta r_{s}=r_{s}(t)-a_{s}$ and $\delta \phi_{s}=\phi_{s}(t)-n_{s} t$. The solid lines are the result of numerical integration of eq1 2 and 3 for $P_{p} / P_{s}=20$, scaled by the factor $\left(P_{p} / P_{s}\right)^{2}$. The dashed lines are the analytic results including the periodic components from eq 10 and 11 as well as the time-independent shift to the radius $\delta r_{s} / a_{s}=\left(n_{p} / n_{s}\right)^{2} / 2$, and the resultant change in orbital period. This change in orbital period is the cause of the linear behavior of $\delta \phi$ in the lower plot, while the oscillatory term from eq[1] causes the short period variations.

The first term on the right hand side of eq 9 gives a shift in the orbital radius $\delta r_{s}=(1 / 2) a_{s}\left(n_{p} / n_{s}\right)^{2}$. This constant term causes no tidal heating, so we ignore it from here on. The second term on the right hand side of eq9 causes an oscillatory change in $\delta r_{s}$. We can solve for this term by writing $\delta r_{s}(t)=\delta r_{s 0} \cos \left(\left[2\left(n_{s}-n_{p}\right) t+2 \phi_{s 0}\right]\right)$, where $\delta r_{s 0}$ is a constant. The cosine factor then cancels out of the equation and the amplitude $\delta r_{s 0}$ can be found. The result is

$$
\frac{\delta r_{s}(t)}{a_{s}}=-\left(\frac{n_{p}}{n_{s}}\right)^{2} \cos \left[2\left(n_{s}-n_{p}\right) t+2 \phi_{s 0}\right] .
$$

Plugging eq 8 and 10 into eq2, we find the angle

$$
\delta \phi_{s}(t)=\frac{11}{8}\left(\frac{n_{p}}{n_{s}}\right)^{2} \sin \left[2\left(n_{s}-n_{p}\right) t+2 \phi_{s 0}\right] .
$$

Eq 10 and 11 confirm that the forced perturbations vary as $\left(P_{s} / P_{p}\right)^{2}$ for small eccentricity orbits. These formulas are valid when $P_{s} \ll P_{p}$, and underestimate the perturbations as the orbit approaches instability. We will use eq 10 and 11 to compute the tidal heating rate in section 4.

Figure 1 compares a direct numerical integration of eq. 1. 2 and 3 against the analytic formulas in eq 10 and 11 showing that they agree to the stated accuracy in the small parameter $P_{s} / P_{p}$.

The discussion so far has examined small perturbations about circular planet and satellite orbits. The same method can be used to find small perturbations, induced by the star, about orbits with finite "background" eccentricities $e_{s 0}$ or $e_{p 0}$. A detailed derivation and results can be found in Brouwer \& Clemence (1961). Again orbital variations of size $\left(P_{s} / P_{p}\right)^{2}$ are found due to stellar perturbations. Tidal heating of the satellite can arise due to either background $e_{s 0}$ and $e_{p 0}$, or perturbations induced by the star, which exists even for $e_{s 0}=e_{p 0}=0$. We show in section 4 that the relative size of these two effects scales as $e_{s 0}$ or $e_{p 0}$ versus $\left(P_{s} / P_{p}\right)^{2}$. The forced variation should provide a lower limit to the tidal heating, and may significantly underestimate the heating for finite eccentricity orbits at large $a_{p}$.

\section{ORBITAL DECAY DUE TO TIDES RAISED IN THE GAS GIANT PLANET}

Tidal friction is recognized to be an important factor in the survival of hypothetical early satellite systems for inner Solar System planets Mercury and Venus (e.g., Burns 1973; Ward \& Reid 1973). These studies pointed out that tides raised in the planet by the satellite and the Sun can cause significant changes in the satellite's orbit. The problem is even more severe as the planet is moved closer to the star, as the stellar tides are stronger and the Hill sphere is closer to the planet. However, a key difference occurs if the planet in question is a gas giant rather than a rocky body. Gas giants are less dissipative than rocky bodies, and hence satellites are relatively more immune to destruction by orbital decay into the planet.

Barnes \& O'Brien (2002) studied orbital decay of satellites around hot Jupiters. They point out that if the gas giant planet is synchronized to the parent star, then the satellite orbits faster than the planet spins. Tides raised in the planet by the satellite then attempt to spin up the planet while shrinking the orbit of the satellite to conserve angular momentum. The conclusion of their study was that only satellites with masses $M_{s}<<M_{\oplus}$ could survive being dragged down to the planet. Such tiny satellites would be undetectable by the transit method. We point out that recent theoretical work on the tidal $Q_{p}$ in gas giants implies that orbital decay is far less effective than the estimate by Barnes \& O'Brien (2002) for the forcing periods of interest, and hence orbital decay may be ineffective even for Earth mass satellites.

Using the equations from Goldreich \& Soter (1966), orbital decay over a timescale $T$ will lead to destruction of satellites with mass

$$
M_{s} \geq M_{s, \text { crit }}=5.4 \times 10^{-3} M_{p}\left(\frac{Q_{p} P_{s}^{13 / 3}}{P_{\mathrm{dyn}}^{10 / 3} T}\right),
$$

where $Q_{p}$ is the quality factor for the gas giant planet and $P_{\mathrm{dyn}}=2 \pi\left(R_{p}^{3} / G M_{p}\right)^{1 / 2}$ is the dynamical time of the planet (2.8 hr for Jupiter). Barnes \& O'Brien (2002) evaluated this expression using $Q_{p} \simeq 10^{5}$, the value inferred for orbital expansion in the Jupiter-Io system (Goldreich \& Soter 1966), as well as circularization of the extrasolar planets (e.g., Wu 2003), finding

$$
\begin{aligned}
M_{s, \text { crit }} & =5 \times 10^{-5} M_{\oplus}\left(\frac{M_{p}}{M_{\mathrm{Jup}}}\right)\left(\frac{Q_{p}}{10^{5}}\right) \\
& \left.\times\left(\frac{P_{s}}{P_{p} / 5} \frac{P_{p}}{4 \text { days }}\right)^{13 / 3}\left(\frac{2.8 \mathrm{hr}}{P_{\mathrm{dyn}}}\right)^{10 / 3}\left(\frac{5 \mathrm{Gyr}}{T}\right) 13\right)
\end{aligned}
$$

where $M_{\text {Jup }}$ is the mass of Jupiter, and we have scaled $P_{s}$ in terms of the maximum orbital period $P_{p} / 5$. If true, this result would imply that Earth mass satellites would only be stable outside orbital periods of $\sim 40$ days, with 
corresponding smaller transit probability than for the hot Jupiters at $P_{p} \sim$ a few days.

The physical origin of the tidal $Q_{p}$ in gas giant planets has been an outstanding question since at least the 1970's. The $Q_{p}$ for Jupiter is constrained to be $Q_{p} \sim 10^{5}-10^{6}$ in order that Io's orbit expanded into the Laplace resonance (Goldreich \& Soter 1966). Early theoretical work by Hubbard (1974) showed that the turbulent viscosity generated by convective eddies required for the outward transport of heat would give rise to $Q_{p} \sim 10^{5}$, perhaps explaining the observed value. Goldreich \& Nicholson (1977) then pointed out that turbulent eddies in the planet have long turnover times, $t_{\text {eddy }}$, compared to the forcing periods of interest, severely decreasing the turbulent viscosity used in Hubbard's calculation. Goldreich \& Nicholson (1977) estimated $Q_{p} \sim 10^{13}$ for "equilibrium-tide" flow in Jupiter, underpredicting the observed tidal dissipation rate by a factor of $10^{7}-10^{8}$. $\mathrm{Wu}(2005)$ revisited Goldreich and Nicholson's calculation, revising $Q_{p}$ downward to $Q_{p} \simeq 10^{12}$.

The detailed physics underlying tidal dissipation in gas giants may strongly affect predictions of the orbital stability of the satellites studied in this paper. Recent calculations by Ogilvie \& Lin (2004) and Wu (2005) have found that when the tidal forcing frequencies are resonant with inertial waves (waves with frequencies less than twice the spin frequency, restored by the Coriolis force) that $Q_{p} \sim 10^{5}-10^{9}$ can be obtained, due to the higher dissipation rate for short lengthscale inertial waves. Conversely, when the forcing frequency is not in the inertial range, no such resonant excitation occurs and $Q_{p}$ will be orders of magnitude larger, comparable to the equilibrium tide value $Q_{p} \sim 10^{12}$, or perhaps somewhat smaller if atmospheric waves can dissipate efficiently. The work of Ogilvie and Lin and $\mathrm{Wu}$ has demonstrated that resonant excitation of inertial waves is a promising mechanism to explain the small observed $Q_{p}$ values. A generic feature in their results is that the tidal dissipation rate is strongly dependent on the forcing frequency. Hence the practice of taking a measured $Q_{p}$ from one situation and applying it to another may be incorrect if the forcing frequencies are very different. The consequences of the tidal dissipation factor varying with forcing frequency have already been explored in the context of solar type stars by Ogilvie \& Lin (2007).

We now explore the consequences for survival of satellites of short period exoplanets if the inertial wave hypothesis is correct. If synchronized, the spin period of the gas giant equals its orbital period, $P_{p}$. But the orbital period of the satellite must satisfy $P_{s} \leq P_{p} / 5$ for orbital stability. For a quadrupole tide raised in the planet, the forcing period would be shorter than $P_{p} / 10$, while inertial waves only exist with periods longer than $P_{p} / 2$. Hence inertial waves cannot be excited in synchronous planets by the satellite and $Q_{p} \sim 10^{5}$ does not apply.

The existence of exomoons around close-in gas giant exoplanets with $P_{p} \lesssim$ a couple months provides a test of tidal dissipation theory. From eq.13 in order for a satellite of mass $M_{s}$ to survive for a time $T$ requires

$Q_{p} \geq Q_{\mathrm{p}, \mathrm{crit}}=2 \times 10^{9}\left(\frac{M_{s}}{M_{\oplus}}\right)\left(\frac{M_{\mathrm{Jup}}}{M_{p}}\right)\left(\frac{P_{p}}{5 P_{s}} \frac{4 \text { days }}{P_{p}}\right)^{13 / 3}$

$$
\times\left(\frac{P_{\mathrm{dyn}}}{2.8 \mathrm{hr}}\right)^{10 / 3}\left(\frac{T}{5 \mathrm{Gyr}}\right) .
$$

This lower limit on $Q_{p}$ is well above the canonical value $Q_{p} \sim 10^{5}-10^{6}$, and thus could confirm the expected large difference in $Q_{p}$ between forcing in and out of the frequency range of inertial waves. Outside the inertial range, the equilibrium tide calculation gives $Q_{p} \sim 10^{12}$ (Goldreich \& Nicholson 1977; Wu 2005), so that Earthsize satellites of hot Jupiters would be immune to destruction by orbital decay over Gyr timescales. Note however, that even if $Q_{p} \sim 10^{5}-10^{6}$, Earth size satellites could exist for longer period orbits $P_{p} \gtrsim$ a couple months.

\section{HEATING OF THE SATELLITES OF CLOSE-IN EXOPLANETS}

This far we have studied two facets of satellite orbits. First the variations induced in the satellite orbit by stellar gravity were reviewed. Then we showed that satellite orbits may be far less susceptible to orbital decay than previously thought if $Q_{p} \gg 10^{5}-10^{6}$ for forcing outside the inertial frequency range, as occurs for a satellite orbit around a synchronized planet. Given that long-lived satellites may exist around even short-period exoplanets, we now investigate the consequences of their orbital variation on tidal heating. To motivate our study, we first review tidal heating in Io.

Based on the significant forced eccentricity of Io, Peale et al. (1979) predicted widespread surface volcanism, which was soon confirmed by images from Voyager 1 (Masursky et al. 1979). The total dissipation rate for tidal forcing of a homogeneous, incompressible, elastic sphere is (Peale \& Cassen 1978; Peale et al. 1979; Wisdom 2004)

$$
\dot{E}=\frac{42}{19} \frac{\pi \rho_{s}^{2} n_{s}^{5} R_{s}^{7} e_{s 0}^{2}}{\mu_{s} Q_{s}}
$$

where $\mu_{s} \simeq 6.5 \times 10^{11}$ dyne $\mathrm{cm}^{-2}$ is the rigidity, and $Q_{s}$ is the tidal dissipation coefficient of Io. For Io, $P_{s}=1.7$ days, $\rho_{s}=3.5 \mathrm{~g} \mathrm{~cm}^{-3}$, and $R_{s}=1800 \mathrm{~km}$. The eccentricity of Io was determined to be $e_{s 0}=0.0043$ (Peale et al. 1979), resulting in a dissipation rate for Io of

$$
\dot{E}_{\mathrm{Io}}=\frac{1.6 \times 10^{21}}{Q_{s}} \mathrm{erg} \mathrm{s}^{-1} .
$$

Peale et al. (1979) used a fiducial value $Q_{s}=100$, based on laboratory studies on rock samples. For $Q_{s}=100$, eq16 gives a heating rate several times the radioactive heating rate estimate for the Moon (Peale et al. 1979). Tidal heating is widely recognized as an important effect in the thermal structure of Io.

To assess the role of tidal heating for the satellites around hot Jupiters, we first ask what would happen to an Io-like satellite orbiting around a Jupiter-like planet, which itself is in 4 day orbit around a solar-type star. From eq 10 and 11, the perturbations correspond to eccentricity variations $\sim\left(P_{s} / P_{p}\right)^{2} \lesssim 1 / 25=0.04$. For orbits near the stability limit, the forced eccentricity is larger than the eccentricity of Io by a factor of $0.04 / 0.0043=9$. The orbital period of the satellite must be smaller than 4 days $/ 5=19 \mathrm{hr}$, shorter than Io's orbital period by a factor of $\simeq 2.1$. Plugging into eq 15 , the 
higher eccentricity and shorter orbital period combine to increase the heating rate by a factor $\sim 10^{4}$. Clearly tidal heating will be important for satellites around hot Jupiters even for satellite size much smaller than Io.

Tidal heating rates for Earth-like satellites are sensitively dependent on the tidal $Q_{s}$. The value $Q_{\text {Earth-Moon }} \sim 10$ for the Earth, as derived from EarthMoon orbital evolution, is likely due to dissipation in shallow seas (e.g. Jeffrevs 1970). Heat from such dissipation depends on the uncertain ocean depth and topography, and is easily radiated away. We focus instead on heating of the interior, especially for early stages to determine if the satellite is melted. Decay of seismic body waves with periods of $10^{3}-10^{4} \mathrm{~s}$ in the Earth finds $Q_{\text {Earth seismic }} \sim 300-500$ (e.g. Roult et al. 2006), but extrapolation of this data to $\sim 10^{5}-10^{6} \mathrm{~s}$ is uncertain (e.g. Burns 1977; Efroimsky \& Lazarian 2000). For the solid phase, we will use a fiducial value of $Q_{s}=100$, assuming moderately increased dissipation when the period is extrapolated over $10-100$ longer timescales than is measured. As we will show, the large tidal heating rates for a solid satellite with $Q_{s} \sim 100$ imply that satellite is likely melted on a short timescale. The subsequent tidal heating rate depends on the appropriate $Q_{s}$ for the liquid state, which is uncertain. For a completely molten satellite, $Q_{s}$ may be much larger. For instance, the Preliminary Reference Earth Model, constructed from seismological data, uses $Q_{s} \sim 10^{5}$ for the Earth's liquid outer core (Dziewonski \& Anderson 1981).

The tidal heating rate in eq 15] derived by Peale et al. (1979) is for the case of a Keplerian satellite orbit with eccentricity $e_{s 0}$. This is only strictly valid for a satellite orbit unperturbed by external influence. Here we are concerned with satellite orbits which may be significantly perturbed by stellar gravity. In other words, we require a tidal heating rate for satellite orbits given by the solution to the restricted 3-body problem (for small satellite mass), rather than for a Keplerian orbit. The orbital variation due to stellar gravity was summarized in section 2 .

To discuss the tidal heating rate for satellites perturbed by stellar gravity we will derive an analytic estimate by plugging eq10 and 11 into the formula found in Wisdom (2004):

$$
\dot{E}=\frac{\rho_{s} h_{s} R_{s}^{2}}{g_{s}} \int d \Omega U \frac{d U^{\prime}}{d t} .
$$

This more general formula allows an arbitrary orbit, and is not specialized to the case of an unperturbed Keplerian orbit. Here $h_{s}=(5 / 2) /\left(1+19 \mu_{s} / 2 \rho_{s} g_{s} R_{s}\right)$, $g_{s}=G M_{s} / R_{s}^{2}, U$ is the tidal potential in the satellite due to the planet, $U^{\prime}$ is the tidal potential including the dissipative lag, and the integral extends over the surface of the satellite. We perform several operations to simplify eq,17. First, we expand $U$ in spherical harmonics and perform the angular integration. Next, we expand to leading order in the lag time $\left(n_{s} Q_{s}\right)^{-1}$. We assume the satellite is synchronized when evaluating the libration term. Lastly, we plug in the small deviations from a circular orbit found in eq[10 and 11. We find the result

$$
\dot{E}=\frac{2817}{160} \frac{G M_{p}^{2} h_{s} R_{s}^{5} n_{s}}{Q_{s} a_{s}^{6}}\left(\frac{n_{p}}{n_{s}}\right)^{4} .
$$

Note the difference between eq.s 15 and 18. Eq15 is

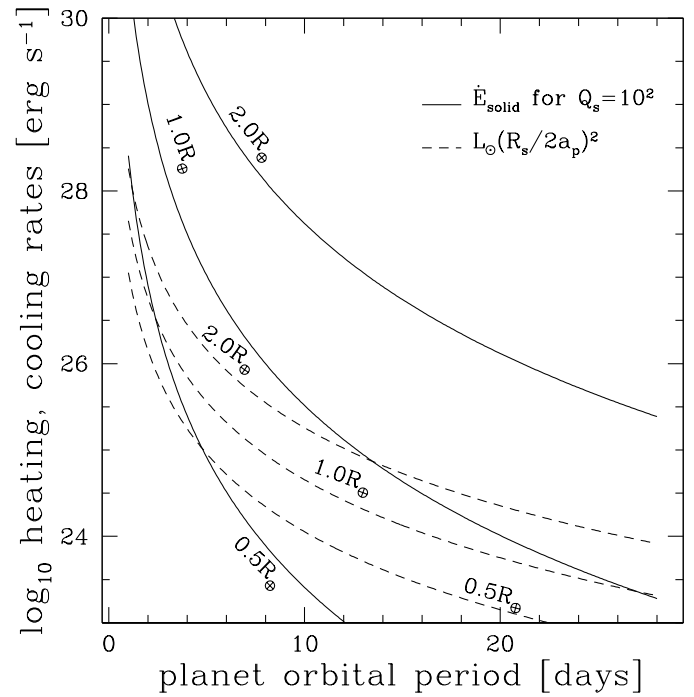

FIG. 2.- Satellite tidal heating rates (solid lines) as a function of orbital period. Eq 19 was used for the heating rate with $\rho_{s}=$ $5 \mathrm{~g} \mathrm{~cm}^{-3}, Q_{s}=10^{2}$ and $P_{s}=P_{p} / 5$. Insolation rates (dashed lines) for a Sun-like star are shown for comparison.

proportional to $e_{s 0}^{2}$, which is assumed constant for the unperturbed Kepler orbit, while eq 15 is proportional to $\left(n_{p} / n_{s}\right)^{4}$, the rms eccentricity squared induced in the orbit by the stellar gravity. No terms involving $e_{s 0}$ appear in eq,18 as we have perturbed around a Keplerian orbit with $e_{s 0}=0$. Alternatively, a more involved calculation would be to perturb around an orbit with finite $e_{s 0}$, in which case presumably the terms in eq[15 and eq[18 would appear summed together. That is, plugging the Keplerian result $\delta r=-e_{s 0} a_{s} \cos \left(n_{s} t\right)$ and $\delta \phi_{s}=2 e_{s 0} \sin \left(n_{s} t\right)$ into eq ${ }_{17}^{3}$ would yield eq 15 . Equating these two formulas we find a critical eccentricity $e_{\mathrm{s}, \text { crit }} \simeq 1.6\left(P_{s} / P_{p}\right)^{2} \lesssim 0.06$ above which the term in eq.15 would dominate and below which the term in eq 18 would dominate. The eccentricity $e_{s 0}$ would depend on the initial conditions as well as subsequent tidal dissipation in the satellite and planet which would act to decrease $e_{s 0}$. Henceforth we ignore $e_{s 0}$ and use the tidal heating rate in eq18. Since we have ignored possible finite $e_{s 0}$, we may underestimate the heating rate if $e_{s 0}$ is large.

To numerically evaluate eq 18 , we again use $\mu_{s} \simeq 6.5 \times$ $10^{11}$ dyne $\mathrm{cm}^{-2}$ for the rigidity, and scale all quantities to Earth values. Since the heating rate increases as $P_{s}$ decreases, we scale expressions to the minimum heating rate using $P_{s}=P_{p} / 5$, finding

$$
\begin{aligned}
\dot{E}_{\text {solid }} & =\frac{3.2 \times 10^{29} \mathrm{erg} \mathrm{s}^{-1}}{Q_{s}}\left(\frac{\rho_{s}}{5 \mathrm{~g} \mathrm{~cm}^{-3}}\right)^{2} \\
& \times\left(\frac{R_{s}}{R_{\oplus}}\right)^{7}\left(\frac{4 \text { days }}{P_{p}}\right)^{5}\left(\frac{P_{p} / 5}{P_{s}}\right)
\end{aligned}
$$

for a solid satellite and

$$
\dot{E}_{\text {liquid }}=\frac{2.7 \times 10^{29} \mathrm{erg} \mathrm{s}^{-1}}{Q_{s}}
$$

3 Equivalently one could use eq 29 which is simpler in practice. 


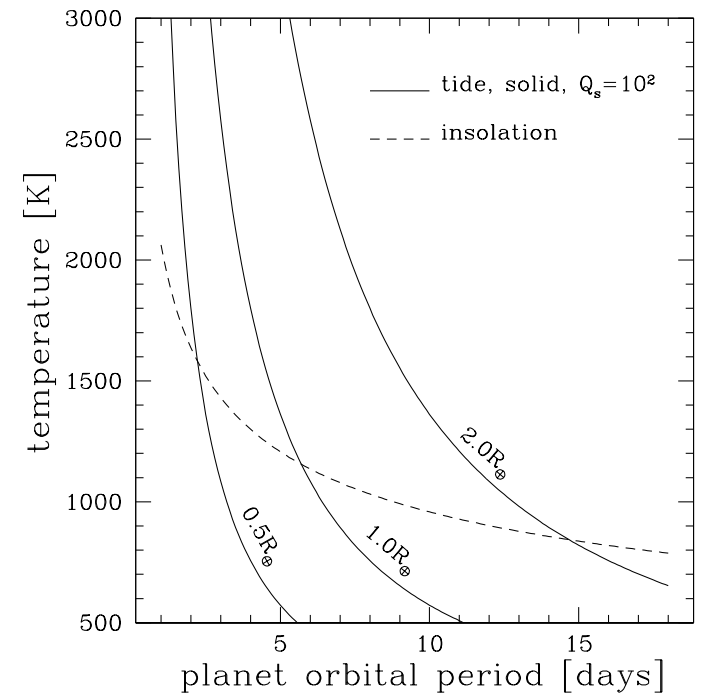

FIG. 3.- Effective temperature due to tidal heating (solid lines) or insolation (dashed line). Solid lines show temperature determined from eq 21 while dashed lines represent the equilibrium temperature due to insolation, and reradiation over $4 \pi$ steradians. Tidal heating determines the temperature when the solid line is above the dashed line.

$$
\times\left(\frac{R_{s}}{R_{\oplus}}\right)^{5}\left(\frac{4 \text { days }}{P_{p}}\right)^{5}\left(\frac{P_{p} / 5}{P_{s}}\right)
$$

for a satellite which has been completely melted $\left(\mu_{s}=\right.$ $0)$. Eq19 is compared to insolation in fig,2. While the prefactors in eq,19 and 20 are comparable, their scaling with $R_{s}$ differs, and the tidal $Q_{s}$ may be quite different for molten and solid satellites. If the tidal $Q_{s}$ in the liquid phase is far larger than for the solid phase, then the rate of tidal heating could drop drastically upon melting.

Would Earth-size satellites around hot Jupiters be melted by tidal heating? Eq,19 implies a heating rate $\dot{E}_{\text {solid }} \sim 10^{28} \mathrm{erg} \mathrm{s}^{-1}$ for a 4 day orbital period. For a latent heat $L=10^{10} \mathrm{erg} \mathrm{g}^{-1}$ appropriate for iron at high pressure (Stevenson et al. 1983), melting would occur in a few hundred years. The tidal heating rate, per gram, scales as $R_{s}^{4}$, so that heating is more intense for larger satellites. Assuming the radioactive heating rate of the Earth, $\sim 10^{20} \mathrm{erg} \mathrm{s}^{-1}$, tidal heating dominates radioactive heating for $P_{p} \lesssim 150$ days. Radioactive heating will eventually dominate for satellites far smaller than an Earth radius. Thermal models of the young Earth (Stevenson et al. 1983) typically show a completely liquid core for $\dot{E} \gtrsim 10^{20} \mathrm{erg} \mathrm{s}^{-1}$; the tidal heating rate is greater by many orders of magnitude. We conclude that the Earth-size satellites of hot Jupiters, observable by their transits, would be largely molten, except perhaps for a thin layer near the surface, cooled by radiation (e.g., Peale et al. 1979). If $Q_{s}$ drops precipitously for complete melting, the temperature could be regulated near the melting point.

The effective temperature for the tidal heating energy flux may be found by equating the heating rate in eq,19 to the blackbody cooling rate $4 \pi R_{s}^{2} \sigma T_{\mathrm{eff}}^{4}$, giving

$$
T_{\text {eff }}=1800 \mathrm{~K}\left(\frac{\rho_{s}}{5 \mathrm{~g} \mathrm{~cm}^{-3}}\right)^{1 / 2}\left(\frac{R_{s}}{R_{\oplus}}\right)^{5 / 4}
$$

$$
\times\left(\frac{4 \text { days }}{P_{p}}\right)^{5 / 4}\left(\frac{P_{p} / 5}{P_{s}}\right)^{1 / 4}\left(\frac{10^{2}}{Q_{s}}\right)^{1 / 4} .
$$

Tidal heating dominates the temperature structure, even at the surface, when the temperature in eq.21 is larger than the equilibrium temperature implied by insolation. Figure 3 compares these temperatures for $Q_{s}=10^{2}$, for a zero-albedo surface with uniform temperature over the entire surface. Clearly for $Q_{s}=10^{2}$, Earth-sized objects are raised above the melting temperature at zero pressure $\left(T_{\text {melt }} \lesssim 2000 \mathrm{~K}\right)$ for either iron or rock compositions inside a critical orbital period, which we estimate as

$$
\begin{aligned}
P_{\mathrm{p}, \text { melt }} & =3.7 \text { days }\left(\frac{2000 \mathrm{~K}}{T_{\text {melt }}}\right)^{4 / 5}\left(\frac{\rho_{s}}{5 \mathrm{~g} \mathrm{~cm}^{-3}}\right)^{2 / 5} \\
& \times\left(\frac{R_{s}}{R_{\oplus}}\right)\left(\frac{10^{2}}{Q_{s}}\right)^{1 / 5} .
\end{aligned}
$$

Hence, at the orbital periods of the hot Jupiters (a few days), satellites Earth size or larger are completely molten for $Q_{s}=10^{2}$.

Vigorous tidal heating has consequences for the satellite magnetic field. Fluid motions in the conducting core driven by tidal heating may generate magnetic fields through dynamo action. Stevenson et al. (1983) derive a magnetic field for the Earth by equating the energy available for dynamo generation to the Ohmic dissipation rate of the field, which yields the scaling $B^{2} \propto \dot{E}$, where $\dot{E}$ is the tidal heating rate. As the dissipation rate in eq 20 is larger than the Earth's cooling rate by a factor $\sim 10^{9} / Q_{s}$, our results imply that fields larger than Earth's can be created if $Q_{s}<10^{9}$. Intrinsic magnetic field can decrease the mass loss rate of the atmosphere. Magnetic interaction between the satellite and planetary magnetosphere may be of the Jupiter-Ganymede type (Kivelson et al. 1997) in which the standoff distance is determined mainly by magnetic stresses.

Studies of habitability of satellites around gas giants (e.g., Reynolds et al. 1987; Scharf 2006; Williams et al. 1997) have invoked tidal heating to maintain plate tectonics, create subsurface oceans, etc. They computed satellite tidal heating for finite eccentricity, assuming it to be a free parameter, or pumped to large values by interaction with another satellite. The mechanism for tidal heating in this paper, periodic eccentricity forced by the star, is likely unimportant for satellite habitability, since it is only large for systems very close to the star, where water is already in liquid or vapor form. Tidal heating is of increasing importance, relative to insolation, for host stars of smaller mass since the stellar luminosity drops rapidly.

\section{ENERGETICS}

Tidal dissipation in the satellite takes energy out of the satellite orbit and deposits it in the satellite body as heat. In this section we show that the energy lost from the satellite orbit by heating is exactly balanced by energy gain from work by the stellar gravity. The tidal friction acts to create a lag in the satellite orbital velocity. This lag has the correct phase to allow energy input to the satellite's orbit at a rate sufficient to power the tidal dissipation in the satellite. Ultimately, the energy reservoir for tidal heating of the satellite must then be the star-planet orbit. 
Tidal friction acts to exert a velocity dependent acceleration on the reduced mass of the satellite-planet system (Mardling \& Lin 2002, eq.4) with radial and angular components given by

$$
\begin{aligned}
& a_{r}^{(\mathrm{TF})}=-\gamma_{0} \dot{r}_{s} \\
& a_{\phi}^{(\mathrm{TF})}=-\gamma_{0} r_{s}\left(\dot{\phi}_{s}-\Omega_{s}\right) .
\end{aligned}
$$

Here $\gamma_{0}=(9 / 5)\left(n_{s} h_{s} / Q_{s}\right)\left(m_{p} / m_{s}\right)\left(R_{s} / a_{s}\right)^{5}$ is the frictional drag coefficient, which is much smaller than the orbital mean motion $n_{s}$. Again we choose a circular background orbit plus small perturbations as in eq 5, 6] and 7. Eq,23] and 24] become

$$
\begin{aligned}
& a_{r}^{(\mathrm{TF})}=-\gamma_{0} \delta \dot{r}_{s} \\
& a_{\phi}^{(\mathrm{TF})}=-\gamma_{0} a_{s}\left(n_{s}-\Omega_{s}+\delta \dot{\phi}_{s}\right) .
\end{aligned}
$$

Assuming that the satellite spin has already reached the synchronous state, we may set $\Omega_{s}=n_{s}$ in eq.26.

Adding the tidal friction acceleration in eq.25] and 26 into eq, 8 and 9, and again ignoring the constant radial force term, we find equations which have the form of a damped, driven oscillator. In the limit $\gamma_{0} \ll n_{s}$ we find the solutions

$$
\begin{aligned}
\frac{\delta \dot{r}_{s}}{a_{s}} & =2\left(\frac{n_{p}^{2}}{n_{s}}\right) \sin \left[2\left(n_{s}-n_{p}\right) t+2 \phi_{s 0}\right] \\
& +\frac{35}{6} \gamma_{0}\left(\frac{n_{p}}{n_{s}}\right)^{2} \cos \left[2\left(n_{s}-n_{p}\right) t+2 \phi_{s 0}\right] \\
\delta \dot{\phi}_{s} & =\frac{11}{4}\left(\frac{n_{p}^{2}}{n_{s}}\right) \cos \left[2\left(n_{s}-n_{p}\right) t+2 \phi_{s 0}\right] \\
& -\frac{173}{24} \gamma_{0}\left(\frac{n_{p}}{n_{s}}\right)^{2} \sin \left[2\left(n_{s}-n_{p}\right) t+2 \phi_{s 0}\right] .
\end{aligned}
$$

The first terms in eq 27 and 28 agree with eq, 10 and 11 The second terms proportional to $\gamma_{0}$ describe a lag in the satellite orbit due to tidal friction.

The energy lost from the orbit is found by computing the work done by the tidal friction force. Since the tidal friction force already contains the small parameter $\gamma_{0} \ll$ $n_{s}$, we can ignore terms of order $\gamma_{0}$ in eq27 and 28. For reduced mass $\mu_{s p}=M_{s} M_{p} /\left(M_{s}+M_{p}\right) \simeq M_{s}$, and time averaging so that $\cos ^{2}, \sin ^{2} \rightarrow 1 / 2$, we find

$$
\frac{\dot{E}_{\mathrm{s}, \mathrm{orb}}^{(\mathrm{TF})}}{\mu_{s p}}=-\gamma_{0}\left(3 \delta \dot{r}_{s}^{2}+a_{s}^{2} \delta \dot{\phi}_{s}^{2}\right)=-\left(\frac{313}{32}\right) \gamma_{0} a_{s}^{2}\left(\frac{n_{p}^{2}}{n_{s}}\right)^{2}
$$

This is just a rederivation of eq,18.

The increase in satellite orbital energy due to work done by the stellar gravitational force in eq 8 and 9 is

$$
\begin{aligned}
\frac{\dot{E}_{\mathrm{s}, \text { orb }}^{\text {(star })}}{\mu_{s p}} & =\frac{3}{2} n_{p}^{2} a_{s}\left(\delta \dot{r}_{s} \cos \left[2\left(n_{s}-n_{p}\right) t+2 \phi_{s 0}\right]\right. \\
& \left.-a_{s} \delta \dot{\phi}_{s} \sin \left[2\left(n_{s}-n_{p}\right) t+2 \phi_{s 0}\right]\right) .
\end{aligned}
$$

The terms independent of $\gamma_{0}$ in eq27 and 28 time average to zero when inserted in eq30. Inserting the terms proportional to $\gamma_{0}$ into eq 30 and time averaging we find

$$
\frac{\dot{E}_{\mathrm{s}, \mathrm{orb}}^{(\mathrm{star})}}{\mu_{s p}}=\left(\frac{313}{32}\right) \gamma_{0} a_{s}^{2}\left(\frac{n_{p}^{2}}{n_{s}}\right)^{2}
$$

exactly equal and opposite to the energy lost to tidal heating in eq29.

We have found that energy lost from the orbit is replenished by work done on the orbit by the stellar tidal force. Hence the satellite orbit is stable on long timescales to tidal effects arising from dissipation in the satellite. The energy reservoir powering the tidal dissipation in the satellite must then come from the star-planet orbit. For star-planet orbital energy

$$
\begin{aligned}
E_{\star \mathrm{p}} & =-\frac{G M_{\star} M_{p}}{2 a_{p}} \\
& =-1.8 \times 10^{44} \operatorname{erg}\left(\frac{M_{\star}}{M_{\odot}}\right)^{2 / 3}\left(\frac{M_{p}}{M_{\text {Jup }}}\right)\left(\frac{4 \text { days }}{P_{p}}\right)^{2 / 3}(32)
\end{aligned}
$$

and dissipation in a liquid satellite (eq20), the starplanet orbit is immune to orbital decay on a timescale $T$ if

$$
\begin{aligned}
Q_{s} & \geq 480\left(\frac{M_{\star}}{M_{\odot}}\right)^{-2 / 3}\left(\frac{M_{p}}{M_{\mathrm{Jup}}}\right)^{-1}\left(\frac{R_{s}}{R_{\oplus}}\right)^{5} \\
& \times\left(\frac{4 \text { days }}{P_{p}}\right)^{13 / 3}\left(\frac{P_{p} / 5}{P_{s}}\right)\left(\frac{T}{10^{10} \mathrm{yr}}\right) .
\end{aligned}
$$

This critical value of $Q_{s}$ for orbital decay is comparable to the value expected for a solid Earth-like satellite (see section (4). In the more likely case that the satellite has been melted by tidal heating and $Q_{s} \gg 10^{2}$, the starplanet orbit is immune to decay.

\section{EVAPORATIVE MASS LOSS}

The large tidal heating rates in eq 19 and 20, large insolation from the parent star, and low escape speed raise the possibility of enhanced rates of mass loss from the satellites studied in this paper. The rates of mass loss are difficult to predict, and may depend on many factors such as stellar wind or magnetospheric erosion of the atmosphere, and the flux of vapor from the surface to the exosphere. Here we discuss one possible scenario, of thermal evaporation from the solid surface of a satellite with a negligible atmosphere (such as the Moon or Mercury).

Cameron (1985) and Fegley \& Cameron (1987) discussed evaporative mass loss from Mercury in the protoplanetary nebula as a means of understanding Mercury's high mean density. The high temperatures in the nebula allow enhanced rates of evaporation of low(er) density silicates from the solid surface into gaseous form. If this vapor is removed from the atmosphere, the mass fraction of iron in the body, and hence the mean density, will increase. Due to the exponential temperature dependence, this process is only effective at high temperatures. We find that for surface temperatures due to tidal heating, or the equilibrium temperature due to insolation, that significant mass loss can occur. We will follow the simple estimate in Cameron (1985) to estimate the importance of this process.

The number flux for thermal evaporation from a surface, in $\mathrm{cm}^{-2} \mathrm{~s}^{-1}$, is given by

$$
\phi=\frac{P_{\mathrm{eq}}(T)}{\sqrt{2 \pi m k_{b} T}},
$$

where $P_{\text {eq }}(T)=P_{0} 10^{-T_{0} / T}$ is the equilibrium vapor pressure above the surface, $m$ is the mean molecular 
weight (in grams) of the products, $T$ is the temperature, and $P_{0}$ and $T_{0}$ describe the equilibrium pressure. Cameron (1985) considers an illustrative example of $\mathrm{MgSiO}_{3}$ (liq) $=\mathrm{Mg}$ (gas) $+\mathrm{SiO}$ (gas) $+\mathrm{O}_{2}$ (gas) (see Fegley \& Cameron (1987) for more detailed calculation of silicate magmas of chondritic composition), for which $P_{0}=10^{13.176}$ dyne $\mathrm{cm}^{-2}, T_{0}=24605 \mathrm{~K}$, and $m=$ $5.5 \times 10^{-23} \mathrm{~g}$. If the surface temperature is near the equilibrium temperature for a zero albedo surface (neglecting tidal heating), radiating with the same efficiency over $4 \pi^{4}$, the equilibrium temperature versus orbital radius for a solar type star is $T=5777 \mathrm{~K}\left(R_{\odot} / 2 a_{p}\right)^{1 / 2}=$ $1300 \mathrm{~K}\left(10 R_{\odot} / a_{p}\right)^{1 / 2}$. As seen in figure 3, this temperature including only insolation, and ignoring tidal heating, may underestimate the satellite surface temperature close to the star. Approximating the exponential as a power law (valid for small changes in $a_{p}$ ), we find a mass flux

$$
m \phi \simeq 1.3 \times 10^{-11} \mathrm{~g} \mathrm{~cm}^{-2} \mathrm{~s}^{-1}\left(\frac{10 R_{\odot}}{a_{p}}\right)^{21.5}
$$

from the surface. If the vapor is lost from the atmosphere, this translates into a mass loss rate

$$
\dot{M}_{s}=3.3 \times 10^{-4} \mathrm{M}_{\oplus} \mathrm{Gyr}^{-1}\left(\frac{10 R_{\odot}}{a_{p}}\right)^{21.5}\left(\frac{R_{s}}{R_{\oplus}}\right)^{2}
$$

In 5 Gyr, an Earth mass can be lost for $a_{p} \lesssim 7.4 R_{\odot}$, where $T \gtrsim 1500 \mathrm{~K}$. Hence satellites originally of Earthsize, and hence detectable by their transit signature with Kepler, may become undetectable due to erosion. Eq 36 implies the rate of decrease of the radius is independent of radius. Erosion does not slow for small satellites.

Our estimates give an upper limit to the erosion rate, and show that large mass loss can occur over the lifetime of the satellite. The exponential dependence on surface temperature suggests a critical orbital radius (of planet around the star) inside of which satellites can be significantly eroded. Our conservative estimate finds the critical orbital period to be around 2.4 days (6.6 days using the day-side equilibrium temperature).

\section{CONCLUSIONS}

4 We note that if the atmosphere is thin (surface pressure $\ll$ 1 bar), the day-side temperature may be larger by a factor of $2^{1 / 2}$. This will cause the critical $a_{p}$ for large mass loss to increase by a factor of 2 , and the critical planet orbital period to increase by a factor $2^{3 / 2}$. Hence a critical orbital period inside of which large
In this paper we have studied the orbits, tidal heating and evaporative mass loss rates from Earth-sized satellites orbiting hot Jupiters. After reviewing the perturbation to satellite orbits due to the stellar gravity, we derived four main results. First, we have shown that orbital decay due to tides raised in the planet by the satellite may be much less efficient that previously thought since gas giants are likely very weakly dissipative at the forcing frequencies of interest. Consequently, even Earthsize satellites may be stable around hot Jupiters over Gyr timescales. Second, large tidal dissipation rates are induced in the satellite due to its forced orbital variations, likely melting all but perhaps a thin surface layer. Third, we found that the satellite orbit does not evolve secularly due to tidal dissipation in the satellite, as the stellar gravity does work at a rate to keep the orbital energy constant. Lastly, the estimated upper limit to mass loss, valid for sufficiently thin atmospheres, indicates a critical orbital period around a Sun-like star (2.4 days for a thick atmosphere, 6.6 days for a thin atmosphere) for Earth-like satellites orbiting a hot Jupiter. Inside this critical period significant erosion can occur, reducing satellites to a size undetectable by upcoming transit observations with the Kepler satellite. Outside the critical period, evaporative mass loss will become negligible so that detectable Earth size planets can survive.

We thank Ken Seidelmann for interesting discussions on lunar theory. TAC was supported by a NASA Graduate Student Researchers Program Fellowship through the Langley Research Center and a Virginia Space Grant Consortium Graduate Research Fellowship. RM received support through a Virginia Space Grant Consortium Undergraduate Scholarship. PA is an Alfred P. Sloan Fellow, and also acknowledges support from the University of Virginia Fund for Excellence in Science and Technology. REJ acknowledges support from NASA's Planetary Atmospheres Program. We thank the anonymous referee for constructive comments which improved this paper significantly.

mass loss occurs depends sensitively on the $2^{1 / 2}$, since it occurs in an exponent. Our assumption of equal day-night temperatures is conservative.

\section{REFERENCES}

Arras, P., \& Bildsten, L. 2006, ApJ, 650, 394

Ballester, G. E., Sing, D. K., \& Herbert, F. 2007, Nature, 445, 511

Barnes, J. W., \& O'Brien, D. P. 2002, ApJ, 575, 1087

Barnes, J. W., \& Fortney, J. J. 2004, ApJ, 616, 1193

Basri, G., Borucki, W. J., \& Koch, D. 2005, New Astronomy Review, 49, 478

Borucki, W., et al. 2004, Stellar Structure and Habitable Planet Finding, 538, 177

Brouwer, D., \& Clemence, G. M. 1961, New York: Academic Press, 1961,

Brown, T. M., Charbonneau, D., Gilliland, R. L., Noyes, R. W., \& Burrows, A. 2001, ApJ, 552, 699

Burns, J. A. 1973, Nature, 242, 23

Burns, J. A. 1977, IAU Colloq. 28: Planetary Satellites,

Cameron, A. G. W. 1985, Icarus, 64, 285

Canup, R. M., \& Ward, W. R. 2006, Nature, 441, 834
Charbonneau, D., Brown, T. M., Noyes, R. W., \& Gilliland, R. L. 2002, ApJ, 568, 377

Domingos, R. C., Winter, O. C., \& Yokoyama, T. 2006, MNRAS, 373,1227

Doyle, L. R., \& Deeg, H.-J. 2004, Bioastronomy 2002: Life Among the Stars, 213, 80

Dziewonski, A. M., \& Anderson, D. L. 1981, Physics of the Earth and Planetary Interiors, 25, 297

Efroimsky, M., \& Lazarian, A. 2000, MNRAS, 311, 269

Fegley, B., \& Cameron, A. G. W. 1987, Earth and Planetary Science Letters, 82, 207

Goldreich, P., \& Soter, S. 1966, Icarus, 5, 375

Goldreich, P., \& Nicholson, P. D. 1977, Icarus, 30, 301

Han, C., \& Han, W. 2002, ApJ, 580, 490

Hubbard, W. B. 1974, Icarus, 23, 42

Jeffreys, H. 1970, Cambridge: University Press, 1970, 
Johnson, R. E., \& Huggins, P. J. 2006, PASP, 118, 1136

Kipping, D. M. 2009, MNRAS, 392, 181 (a)

Kipping, D. M. 2009, MNRAS, 396, 1797 (b)

Kivelson, M. G., Khurana, K. K., Coroniti, F. V., Joy, S., Russell, C. T., Walker, R. J., Warnecke, J., Bennett, L., \& Polanskey, C. 1997, Geophys. Res. Lett., 24, 2155

Mardling, R. A., \& Lin, D. N. C. 2002, ApJ, 573, 829

Masursky, H., Schaber, G. G., Soderblom, L. A., \& Strom, R. G. 1979, Nature, 280, 725

Ogilvie, G. I., \& Lin, D. N. C. 2004, ApJ, 610, 477

Ogilvie, G. I., \& Lin, D. N. C. 2007, ApJ, 661, 1180

Peale, S. J., \& Cassen, P. 1978, Icarus, 36, 245

Peale, S. J., Cassen, P., \& Reynolds, R. T. 1979, Science, 203, 892

Peale, S. J. 1999, ARA\&A, 37, 533

Pont, F., et al. 2007, A\&A, 476, 1347

Redfield, S., Endl, M., Cochran, W. D., \& Koesterke, L. 2008, ApJ, 673, L87

Reynolds, R. T., McKay, C. P., \& Kasting, J. F. 1987, Advances in Space Research, 7, 125

Roult, G., Rosat, S., Clévédé, E., Millot-Langet, R., \& Hinderer, J. 2006, Journal of Geodynamics, 41, 345
Sartoretti, P., \& Schneider, J. 1999, A\&AS, 134, 553

Scharf, C. A. 2006, ApJ, 648, 1196

Simon, A., Szatmáry, K., \& Szabó, G. M. 2007, A\&A, 470, 727

Stevenson, D. J., Spohn, T., \& Schubert, G. 1983, Icarus, 54, 466

Szabó, G. M., Szatmáry, K., Divéki, Z., \& Simon, A. 2006, A\&A, 450,395

Vidal-Madjar, A., Lecavelier des Etangs, A., Désert, J.-M.,

Ballester, G. E., Ferlet, R., Hébrard, G., \& Mayor, M. 2003, Nature, 422, 143

Vidal-Madjar, A., et al. 2004, ApJ, 604, L69

Ward, W. R., \& Reid, M. J. 1973, MNRAS, 164, 21

Williams, D. M., Kasting, J. F., \& Wade, R. A. 1997, Nature, 385, 234

Wisdom, J. 2004, AJ, 128, 484

Wu, Y. 2003, Scientific Frontiers in Research on Extrasolar Planets, 294,213

Wu, Y. 2005, ApJ, 635, 688 